\title{
Ein Dialog mit der Vergangenheit des japanischen Strafrechts
}

\author{
HONDA Minoru*
}

\section{Einleitung}

In seinem Werk Entwicklung und Aufgaben der japanischen Strafrechtswissenschaft analysiert Hirofumi Uchida die moderne japanische Strafrechtsgeschichte anhand von drei Gesichtspunkten. Indem er die Geschichte der Strafgesetzgebung, der strafrechtlichen Präzedenzfälle und der Strafrechtstheorie zueinander in Beziehung setzt, verweist er auf dringende, von der Strafrechtswissenschaft bisher ungelöst gebliebene Aufgaben. Hierzu zählen die theoretische Ausrichtung von Strafrechtsreform und Strafgesetzgebung, basierend auf den Grundsätzen der Verfassung und internationalen Menschenrechtsbestimmungen, sowie die Kritik an den sich hiervon entfremdenden Strafprozessfällen sowie Strafgesetzgebung, und ferner die Errichtung eines demokratischen Strafrechts, das der Doktrin der „bürgerlichen öffentlichen Sicherheit“ (Shimin-teki chian-shugi) ${ }^{1)}$ und einer Überflutung durch Strafgesetzgebung entgegenzutreten vermag. All dies folgt aus einer Analyse der Realität des Strafrechts der Zeit vor dem Krieg sowie deren theoretischer Einordnung und erfüllt für die gegenwärtige und zukünftige theoretische Positionierung der japanischen Strafrechtswissenschaft die Funktion eines Kompasses. Im zweiten Abschnitt mit dem Titel „Die Entwicklung der Strafrechtswissenschaft im Japan der Vorkriegszeit, 5. Faschistische Periode“ aus dem vierten Kapitel „Die Entwicklung der japanischen Strafrechtswissenschaft" werden die Arbeit an einer umfassenden Reform des Strafrechts und die „Bewegung für japanische Rechtsvernunft“ (Nihon Hōri Undō) ${ }^{2)}$ als wichtige Betrachtungsgegenstände genannt. Dies dient vor allem der Feststellung, ob die verfas-

* Professor, Faculty of Law, Ritsumeikan University.

1) Shimin-teki chian-shugi 市民的治安主義: Shimin-teki (bürgerlich) bezieht sich auf den Begriff des Bürgerstrafrechts, chian auf das Strafrecht der öffentlichen Sicherheit (Chian Keihō). Letzteres bezeichnet in Anklang an das Gesetz zur Aufrechterhaltung der öffentlichen Sicherheit (Chian Iji Hō) eine Strafrechtspolitik, die vor allem auf Kontrolle und Unterdrückung setzt und dem Bürger immer weniger Rechte zugesteht. Uchida zufolge sei das Strafrecht der Nachkriegszeit, welches sich nach den demokratischen und freiheitlichen Prinzipien der Verfassung richten sollte, untergraben von der chianDoktrin in Form von strenger Kontrolle durch die öffentliche Verwaltung.

2) Nihon Hōri Undō 日本法理運動: Hōri stellt einen eher ungebräuchlichen Begriff dar, der auch mit „Rechtsprinzip“ oder „Rechtsgrundsatz“ übersetzt werden könnte, für den hier aber aufgrund seiner Unbestimmtheit die Übersetzung „Rechtsvernunft“ gewählt wurde. 
sungs- und menschenrechtlichen Bestimmungen, die das Strafrechtssystem der Vorkriegszeit und dessen Gedankengut in seiner Gesamtheit ablehnen, nach dem Krieg in der japanischen Strafgesetzgebung, den Strafprozessfällen und der Strafrechtswissenschaft verankert wurden, bzw. wie weit man davon noch entfernt ist.

So bleibt Uchida zufolge auch in anderen Rechtsgebieten außerhalb des Strafrechts eine Vielzahl an Fragen bezüglich der Einordnung der Bewegung für japanische Rechtsvernunft als Vertreter einer faschistischen Strafrechtstheorie noch immer unzureichend beantwortet. ${ }^{3)}$ Dieser Beitrag macht es sich zum Ziel, den Gründen hierfür nachzugehen. Die bisher unzureichende theoretische Einordnung der Bewegung für japanische Rechtsvernunft deutet darauf hin, dass auch in der gegenwärtigen Strafrechtswissenschaft die Gefahr des Wiederauflebens eines faschistischen Strafrechts nicht beseitigt ist. Noch heute sieht sich die japanische Strafrechtswissenschaft der Bedrohung durch Totalitarismus und Faschismus gegenüber. Sollte sich die japanische Strafrechtswissenschaft noch nicht aus dieser Starre befreit haben, muss dies zuerst geschehen, bevor man sich den von Uchida aufgeworfenen Fragen zuwendet. Hierzu muss man sich zunächst mit jenem verhängnisvollen Gedankengut, welches die japanische Strafrechtswissenschaft in ihrer Weiterentwicklung hemmt, auseinandersetzen.

\section{Verortung des Themas}

Angesichts der Erlassung des Gesetzes zur Aufrechterhaltung der öffentlichen Sicherheit (Chian Iji Hō), welches den Kokutai ${ }^{4}$ als höchstes zu schützendes Rechtsgut proklamierte, der Verkündung des „Grundrisses einer Strafrechtsreform“ und der Aufnahme der Arbeit an einer Strafrechtsreform, welche die vollkommene Rückkehr zum TennōSystem mit direkter Herrschaft des Kaisers anstrebte, forderte Yazoji Kazahaya inmitten dieser „Verfinsterung des Zeitalters“ als schärfster Kritiker bösartiger Gesetze die umgehende Wiederaufnahme des ,nulla poena sine lege“-Grundsatzes und wurde so Opfer des Gesetzes zur Aufrechterhaltung der öffentlichen Sicherheit. Trotz allem gab Kazahaya nicht auf, und begann stattdessen mit der Übersetzung und Herausgabe von Beccarias Dei delitti e delle pene (,Von den Verbrechen und von den Strafen“) und setzte sich für die Verbreitung des „nulla poena sine lege“-Grundsatzes als Widerstandsgedanken ein. Zur selben Zeit vertrat Yukitoki Takigawa in seinem Strafrechtslesebuch eine liberalistische

3) Vgl. Hirofumi Uchida, Nihon Keihōgaku no Ayumi to Kadai [Entwicklung und Aufgaben der japanischen Strafrechtswissenschaft] (Nihon Hyōronsha, 2008), S. 145 ff. Vgl. hierzu außerdem die Rezension von Minoru Honda, „Rekishi to Keihōgaku“ [Geschichte und Strafrechtswissenschaft], Ritsumeikan Hōgaku Nr. 326 (2010), S. 833 ff.

4) Kokutai 国体: etwa „Staatskörper“, auch „Staatswesen“. Dieser Begriff wurde vor allem in der Zeit ab Ende des 19. Jh. bis zum Zweiten Weltkrieg als wesentlicher Bestandteil der Herrschaftsideologie des jap. Kaiserreiches genutzt und bezeichnet in diesem Zusammenhang die Vorstellung des Staates als einer Art Familie (oder Organismus) mit dem Kaiser an der Spitze. 
Strafrechtstheorie, aus welchem Grund er gezwungen wurde, die Universität zu verlassen. Doch auch Takigawa verteidigte weiterhin den „nulla poena sine lege“-Grundsatz und sprach sich für das Analogieverbot sowie für die Bedeutung von gesellschaftlichen und wirtschaftlichen Faktoren in der Diskussion um die Ursachen für Verbrechen aus. Indem er außerdem die Art der Ausübung der staatlichen Strafgewalt kritisierte, leistete er Widerstand gegen eine Zeit, in der sich Denken und freie Meinungsäußerung in der Krise befanden. Um den Kokutai und das Ie-seido ${ }^{5)}$ vor den immer heftiger werdenden Bewegungen der Bauern- und Arbeiterklasse sowie vor systemfeindlichem Gedankengut zu schützen, wurde eine Ausweitung des Strafsystems zur Unterdrückung dieser angestrebt. Dass inmitten einer solchen Zeit die Bemühungen, eine fortschrittliche, liberalistische Strafrechtstheorie in die Tat umzusetzen, fortgeführt wurden, stellt einen für die japanische Strafrechtsgeschichte besonders erwähnenswerten Umstand dar. Allerdings schwammen viele Strafrechtswissenschaftler mit dem Strom, indem sie das System unterstützten. Sie zeigten sich opportunistisch, unterstützten den „Großostasiatischen Krieg“ und das absolutistische Tennō-System, und passten ihre eigenen strafrechtstheoretischen Positionen an die Ideologie des Kokutai und die herrschende Kriminalpolitik an. Hier boten die politischen Umstände einen Anblick, der vom Idealzustand sehr weit entfernt war. Dies mag man gedanklicher Unsicherheit zuschreiben; allerdings gab es auch Menschen, die erkannt hatten, dass es in der eigenen Verantwortung liegt, zu versuchen, von selbst zu einer Erkenntnis über die Situation zu gelangen. Und auch nachdem die Bewegungen der Bauern- und Arbeiterklasse zerschlagen worden waren, gab es Menschen, die sich einig waren, man müsse als Intellektueller versuchen, mit dem letzten Widerstand des Gewissens den Schaden möglichst in Grenzen zu halten, und die in dieser Situation ebenso großen Einsatz zeigten. Diese Anpassung an die politischen Umstände fand in unterschiedlichen Ausprägungen und aus verschiedenen Gründen statt, jedoch waren es nicht wenige Strafrechtswissenschaftler, die fortan das Strafrecht des japanischen Kaiserreichs lehrten und zur Bewegung für japanische Rechtsvernunft beitrugen. Wie aber konnte es dazu kommen ? ${ }^{6)}$

Zwar führt Uchida den Namen Seiichirō Ono an, welcher sich bewusst für die Bewegung für japanische Rechtsvernunft einsetzte und diese als einer der zentralen Strafrechtswissenschaftler anleitete. Den Namen von Chihiro Saeki allerdings, der sich etwas später der Bewegung für japanische Rechtsvernunft anschloss und sich mit Themen wie „Das Selbstverständnis japanischer Dinge im Strafrecht“ oder „Die im Strafrecht

5） Ie-seido 家制度: Wörtl. „Haussystem“. Hierbei handelt es sich um die traditionelle Organisationsform von Familienverbänden in der japanischen Gesellschaft, mit festen hierarchischen Strukturen und einem Hausherrn an der Spitze. Heute hat sich dieses System weitestgehend gelockert.

6) Eine brillante Darstellung der unglücklichen Situation, in der sich die Intellektuellen in Japan vor und während des Krieges befanden, findet sich bei Ikuo Arakawa, Shōwa Shisō-shi [Geschichte des Denkens der Shōwa-Zeit] (Asahi Sensho, 1989). Der vorliegende Beitrag stützt sich in einigen gedanklichen Ansätzen auf die Analyse Arakawas. 
ersichtliche japanische Tradition" beschäftigte, findet keine Erwähnung. Auch der Name Kazahaya wird in der Strafrechtsgeschichte der Zeit nach der Bewegung für japanische Rechtsvernunft außen vor gelassen. Im Zuge des Kyūdai-Zwischenfalls ${ }^{7)}$ wurde Kazahaya aus seiner Lehrposition entfernt; er übte Kritik am Gesetz zur Aufrechterhaltung der öffentlichen Sicherheit und verteidigte den „nulla poena sine lege“-Grundsatz, woraufhin er als Wissenschaftler innerhalb des „Shōwa Forschungsverbandes“ (Shōwa Kenkyū Kai) einen Artikel mit dem Titel „Der Weg zur Herstellung der Ordnung auf der Grundlage von Wissenschaft und Volk“ veröffentlichte. Entgegen dem Eindruck, den der Titel dieser Abhandlung erwecken mag, maß Kazayaha der Idee einer „Ostasiatischen Gemeinschaft“ eine fortschrittliche Bedeutung bei und bot eine rationale Begründung für die Art und Weise, in der sich das Japanische Reich auf dem chinesischen Festland ausbreitete. ${ }^{8)}$ Erst nach Ende des Zweiten Weltkrieges wurde man auf die Namen dieser beiden Männer aufmerksam. Als Strafrechtstheoretiker der Zeit vor dem Krieg lag ihnen in der Nachkriegszeit daran, ihr Denken von damals fortzuführen und weiterzugeben. Ungeachtet des Erlasses einer neuen Verfassung wurde aufgrund der unangemessenen Ausübung von staatlicher Polizei- und Strafgewalt in einer politisch angespannten Situation, in welcher man beabsichtige, das „Strafrecht der öffentlichen Sicherheit“ (Chian Keihō) wiedereinzusetzen und $\mathrm{zu}$ verschärfen, nach einer widerstandsfähigen Strafrechtstheorie zum Schutz von Menschenrechten verlangt. Die Strafrechtstheoretiker der Vorkriegszeit traten nun als alte Verfechter dieser Anschauung in Erscheinung. Der Grundsatz der strafbaren Rechtswidrigkeit sowie der der Zumutbarkeit, welche in der Nachkriegszeit eine bedeutende Rolle spielten, indem beispielsweise im Zuge der Arbeiterbewegungen der Nachkriegszeit die Rechte der Arbeiter und das Leben des einfachen Volkes vor der gewaltsamen Unterdrückung durch die Kapitaleigner geschützt wurden, sind laut Uchida der Strafrechtslehre Saekis aus der Zeit vor dem Krieg zu verdanken. Weiterhin sieht Uchida den japanischen Ursprung des „nulla poena sine lege“Grundsatzes, welcher die gedankliche Grundlage von Freiheit und subjektiven Rechten darstellt, nicht bei der von Takigawa nach dem Kyōdai-Zwischenfall ${ }^{9)}$ entwickelten Theorie, sondern konstatiert, dass dieser in der bereits zuvor von Kazahaya und Saeki

7) Kyūdai jiken 九大事件：1927 wurden fünf Professoren der juristisch-philosophischen Fakultät der Kyūshū-Universität von ihrem Dienst suspendiert.

8) Yasoji Kazahaya, „Kagaku to Kokumin to no Kiso ni tatsu Jikyoku Shūshū e no Michi“ [Der Weg zur Herstellung der Ordnung auf der Grundlage von Wissenschaft und Volk], in: Kōron, NeujahrSonderausgabe Januar (1940) 126 ff. Eine Analyse der Grundzüge von Kayahazas Forschungstätigkeit vor und während des Krieges bietet Michitoshi Takabatake, „Seisan-ryoku Riron - Ōkōchi Kazuo, Kazahaya Yasoji“ [Theorien der Produktionskraft. Kazuo Ōkōchi und Yasoji Kazahaya], in: Shisō no Kagaku Kenkyū-kai (Hrsg.), Kyōdō Kenkyū Tenkō (chū) [Gemeinschaftsforschung — Die Wende (Bd. 2)] (Heibōsha, 1960), S. $226 \mathrm{ff}$. Jedoch befasst sich diese Darstellung nicht mit Kazahaya als Strafrechtstheoretiker.

9) Kyōdai jiken 京大事件: 1933 wurde Takigawa wegen Vermittlung vermeintlich marxistischem Gedankenguts der Kaiserlichen Universität Kyōto verwiesen. 
aufgebauten Forschung zu suchen sei. ${ }^{10)}$ Jedoch hielten sich sowohl Saeki mit seinen Worten und Taten in Bezug auf die Strafrechtswissenschaft innerhalb der Bewegung für japanische Rechtsvernunft als auch Kazahaya mit seinen Versuchen zur Umsetzung seiner Theorie bezüglich der Idee der „Ostasiatischen Gemeinschaft“ nie zurück. Wenn man nun argumentiert, dass sie trotz alledem in Erwartung des Kriegsendes bereits heimlich an jener Strafrechtslehre arbeiteten, nach der in der Nachkriegszeit verlangt werden sollte, so stellt dies vermutlich nur eine von vielen möglichen Sichtweisen in Bezug auf die Geschichte der Strafrechtswissenschaft dar.

Geht man allerdings nach diesem Verständnis der Strafrechtsgeschichte, so beschränkt man sich bei der kritischen Betrachtung der Strafrechtstheorie des faschistischen Japans vor dem Krieg vor allem auf die fanatische Strafrechtslehre Onos, welcher die Bewegung für japanische Rechtsvernunft anführte. Ein Verständnis der Geschichte des Strafrechts, das von als moderat angesehenen Strafrechtslehren und der Kokugaku-Schule ${ }^{11)}$ geprägt ist, bleibt dabei außer Acht. Onos strafrechtswissenschaftliche Schriften wurden auch Gegenstand der Kritik, weil sie im Widerspruch zum Wertesystem der Verfassung standen, während andere Theorien nicht unmittelbar kritisch betrachtet wurden und im Aufräumprozess innerhalb der Strafrechtsgeschichte unbeachtet bleiben. ${ }^{12)}$ Es verwundert nicht, dass man, statt sich mit solch heiklen Aufgaben zu befassen, der Frage danach Vorrang gab, welche Elemente der Strafrechtswissenschaft vor dem Krieg mit dem Wertesystem der Verfassung in Verbindung standen, um diese als Grundlage der Strafrechtswissenschaft der Nachkriegszeit festzulegen. In der Zeit direkt nach Kriegsende gab es eine kurze Phase der demokratischen Belebung, während der sich um die auch in der „Verfinsterung des Zeitalters" kompromisslos in die Tat umgesetzte politische Theorie der Revolution und deren Geist der Unbeugsamkeit jugendliche Energie zu sammeln begann. Dass man die Weiterführung von Onos Strafrechtslehre zu dieser Zeit ablehnte, scheint selbstverständlich, und es ist offensichtlich, dass man sich der Existenz einer weiteren Kontinuität in der japanischen Strafrechtswissenschaft bewusst wurde, die der Fortführung bedurfte. Dies mag daher kommen, dass durch diese Art von Verständnis eine emotional aufgeladene Stimmung geschaffen wurde, in der sich ebendiese Kontinuität, der es nachzugehen galt, und die Zielstrebigkeit jenes Geistes der Unbeugsamkeit miteinander vermischten. Weiterhin machte es sich die strafrechtsgeschichtliche Forschung zur Aufgabe, die gedanklichen Grundlagen der Strafrechtswissenschaft nach dem Krieg festzulegen und begann, dieser weiteren Kontinuität nachzugehen. Uchida sieht diese Kontinuität nicht etwa in der Strafrechtslehre Takigawas, welcher eine „triumphale Rückkehr“ an die

10) Uchida (Fn. 3), S. 159 ff.

11) Kokugaku 国学: Wörtl. „Landesschule“. Eine seit dem 17./18. Jahrhundert unter verschiedenen Namen bestehende Denkschule, deren Vertreter sich von den chinesischen konfuzianischen Lehren abkehrten und statt derer eine Rückbesinnung auf die eigene indigene Kultur und deren Werte forderten.

12) Vgl. Akira Maeda, Jenosaido-ron [Über den Genozid] (Aoki Shoten, 2001), S. 243 ff. 
Universität Kyōto feiern konnte, sondern in der Lehre Saekis, dem jungen Fahnenträger der Bewegung für japanische Rechtsvernunft. Es fragt sich, ob nicht gerade in dieser Art Verständnis der Hauptgrund für die verspätete kritische Auseinandersetzung mit der Bewegung für japanische Rechtsvernunft liegt.

\section{Die Bewegung für japanische Rechtsvernunft und die Strafrechtslehre Saekis}

Der Band Saekis und Onos Forschungen zur ,japanischen Rechtsvernunft “ von Nakayama Ken'ichi beinhaltet die Aufsätze „Saekis Strafrechtslehre und die ,japanische Rechtsvernunft““ sowie „Eine weitere Untersuchung zu Onos ,Die bewusste Entwicklung der japanischen Rechtsvernunft““, in denen Nakayama die von den beiden Strafrechtswissenschaftlern vor dem Krieg gepredigte „,japanische Rechtsvernunft“ originalgetreu wiedergibt und sie nach heutigen Maßstäben bewertet. Nakayama erörtert, auf welche Weise sich die Strafrechtsforschung an das Staatssystem angepasst und bei der „,japanischen Rechtsvernunft" angelangt war, indem er die Arbeiten der beiden Strafrechtswissenschaftler, die sich den ,ernsten Verhältnissen der Zeit“ gebeugt hatten, unmittelbar zur Diskussion stellt. Dies diene einer erneuten Überprüfung der Bedeutung der Aussage, in der Nachkriegszeit habe keine tiefgreifende Wende im Strafrecht stattgefunden. ${ }^{13)}$ Deshalb besieht sich Nakayama „erneut“ Saekis theoretische Überlegungen zur „japanischen Rechtsvernunft“ in „Saekis Strafrechtslehre und die ,japanische Rechtsvernunft““; jedoch stellte dies mitunter auch „eine Art Tabu“ dar, welches bisher noch nicht direkt angesprochen worden war.

Nakayama selbst hatte bereits in Keihō no Kihon Shisō [Grundgedanken des Strafrechts] stellvertretende Strafrechtswissenschaftler der Vorkriegszeit wie Eiichi Makino oder Yukitoki Takigawa zusammen mit der Entwicklung des Strafrechtsdenkens Saekis vor und nach dem Krieg untersucht und deren Bedeutung geordnet zusammengefasst, ${ }^{14)}$ doch der Grund für eine „erneute“ Untersuchung dieser Bedeutsamkeit findet sich in den folgenden Ereignissen. Nakayama hatte nämlich in Der Takigawa-Zwischenfall ${ }^{15)}$ von Takayoshi Matsuo gelesen, dass Saeki einen Protestbrief gegen die Anordnung zur Entlassung von ungeeignetem Personal an der Universität Kyōto geschrieben und damit selbst eine eigene Beurteilung der Bewegung für japanische Rechtsvernunft abgegeben hatte. Daraufhin entschloss Nakayama sich, „ein weiteres Mal das in Dr. Saekis Werk der

13) Ken“ichi Nakayama, Saeki, Ono-hakase no ,Nihon Hōri' no Kenkyū [Saekis und Onos Forschungen zur ,japanischen Rechtvernunft'] (Seibundō 2011), S. 2.

14) Ken'ichi Nakayama, Keihō no Kihon Shisō [Grundgedanken des Strafrechts] (Seibundō 2003), S. $107 \mathrm{ff}$.

15) Vgl. Takayoshi Matsuo, Takigawa Jiken [Der Takigawa-Zwischenfall] (Iwanami Gendai Bunko 2008), S. $272 \mathrm{ff}$. 
Vorkriegszeit enthaltene Strafrechtsdenken und dabei insbesondere die sogenannte , japanische Rechtsvernunft‘ diesbezüglich zu verfolgen, und darüber hinaus den Zusammenhang zu den ,Geheimnissen' seines Wandels nach dem Krieg zu untersuchen.“ Gleichwohl sollte dies nicht dazu dienen, „einen Teil der Gestalt des Werks Dr. Saekis aus der Zeit vor dem Krieg, welcher längst als veraltet betrachtet und beiseitegelegt wurde" zu enthüllen, sondern „das Erbe Dr. Saekis, der unter den Verhältnissen der Vorkriegszeit gelitten hatte“ offen zu klären, um so „seine Anpassung an die ernsten Verhältnisse der Zeit unmittelbar zu hinterfragen und erneut die Bedeutung seines Wandels nach dem Krieg herauszustellen“. ${ }^{16)}$ Auch die heutige Strafrechtswissenschaft befindet sich offensichtlich in einer zunehmend ernsteren Situation, wenn auch in einem anderen Sinne als zu der Zeit der Bewegung für japanische Rechtsvernunft. Wenn man eine Lehre aus dem ziehen kann, worunter Saeki in den „ernsten Verhältnissen der Zeit“ gelitten hatte, inwiefern dies der Situation zu jener Zeit entsprach und auf welche Weise er darauf reagierte oder nicht reagierte, wird dies wohl dabei hilfreich sein, die grundlegenden Positionen der heutigen Strafrechtswissenschaft zu prüfen. Dies wird auch für die Bewältigung der von Uchida angesprochenen Aufgabe, das Wiederaufleben faschistischer Strafrechtstheorien aus dem Japan der Vorkriegszeit zu verhindern, von großer Bedeutung sein.

Gegenstand von Nakayamas Analyse und Einschätzung ist Saekis Werk ab 1943 (Shōwa 18). Seine Betrachtung umfasst die Aufsätze „Keihō ni okeru Nihon-teki naru mono no Jikaku $(1,2)^{\text {“ }}$ [Das Bewusstsein für japanische Dinge im Strafrecht (Bd. 1 u. 2)] (Hōgaku Ronsō Bd. 49, Nr. 1-2; 1943), „Sekinin-ron ni okeru Nihon-teki Hansei“ [Die japanische Reflexion in der Schuldlehre] (Hōritsu Jihō Bd. 15, Nr. 6; 1943), „Kokugaku no Dentō to Nihon Keihō no Riron“ [Die Tradition der Kokugaku-Schule und die Theorie des japanischen Strafrechts] (Keisei Bd. 57, Nr. 1; 1944) und „Keijihō yori mitaru Nihonteki Dentō" [Die im Strafrecht ersichtliche japanische Tradition] (Hōgaku Ronsō Bd. 50, Nr. 5-6; 1944) sowie das Grundlagenwerk Keihō Sōron [Strafrecht Allgemeiner Teil] (Kōbundō-Verlag; 1944), welches die theoretische Position erkennen lässt, bei der Saeki vor dem Krieg angelangt war. Nach einer sorgfältigen Lektüre dieser Werke stellt Nakayama die Einschätzung Takayoshi Matsuos dazu vor und erläutert darüber hinaus „erneut“ seinen eigenen Standpunkt. Matsuo zufolge sei Saeki in Strafrecht Allgemeiner Teil für den „Versuch, zu begreifen, wie das Wesen des Strafrechts in unserem Lande entstand, und ferner, wie es sich entwickelte“ eingetreten und habe als das japanische Strafrecht durchdringende Prinzipien eine „Tradition der Barmherzigkeit“ sowie den „Grundsatz des Vertrauens“ angeführt. Matsuo sah darin „,keine Anklänge an Ultranationalismus oder Militarismus“. Weiterhin zeigt sich Matsuo in seiner Betrachtung von Saekis Äußerungen zur „Errichtung eines dem Großostasiatischen Krieg angemessenen überragenden japanischen Strafrechts“ und seiner Schriften „Das Bewusstsein für

16) Nakayama (Fn. 13), S. 2. 
japanische Dinge im Strafrecht“ und „Die im Strafrecht ersichtliche japanische Tradition“ eher zurückhaltend: „Hier zeigt sich zwar eine starke nationalistische Tendenz, aber ob man dies als ,Ultra'-Nationalismus bezeichnen kann, ist fraglich“. ${ }^{17)}$ Nachdem er Matsuos Einschätzung wiedergegeben hat, erläutert Nakayama im Weiteren seinen eigenen Standpunkt: „Im Großen und Ganzen stimme ich dieser Aussage zu, möchte jedoch noch einige Punkte hinzufügen“. ${ }^{18)}$ Von Belang sind hier zwei Aspekte, auf die Nakayama in Bezug auf die Verneinung eines „ultranationalistischen“ Charakters des Strafrechtsdenkens Saekis in der Zeit vor dem Krieg hinweist.

Zum Einen hieße es, dass Saeki in seiner Forschung zum japanischen Recht „im Gegensatz zu den derzeitigen Tendenzen, die sich blind am nationalsozialistischen Vorbild orientierten und harte Bestrafungen forderten“, das Ziel verfolgte, ,aufzuzeigen, dass die japanische Tradition der Wahrheit auf Selbstbestimmung, Nachsicht, Offenheit, Vernunft und Bedachtsamkeit beruhe“. Laut Nakayamas Auffassung sei dies eine „positive Beurteilung wert“. Was genau diese „positive Beurteilung“ beinhaltet, wird nicht geklärt. Man erfährt jedoch Weiteres, wenn man seine zuvor in Grundgedanken des Strafrechts geäußerte Einschätzung ${ }^{19)}$ hinzu zieht.

Vor dem Krieg verstärkte sich die Tendenz innerhalb der japanischen Strafrechtswissenschaft, blind dem Strafrecht der Nationalsozialisten nachzueifern. Die Kieler Schule beispielsweise lehnte die mit der Tatbestandslehre verbundene Denkweise ab und verwarf aus einer ganzheitlichen Betrachtungsweise heraus eine abstrakte Unterscheidung von Rechtswidrigkeit und Schuld. Saeki stellte sich dem entgegen, indem er am traditionellen Verbrechensaufbau festhielt; zur gleichen Zeit nahm er eine Position ein, die die von den Nationalsozialisten gepredigte Vorstellung des konkret Allgemeinen in Teilen befürwortet, da dessen grundlegende Annahmen aufschlussreiche Aspekte beinhalteten, welche ein Nachdenken über das herkömmliche Strafrecht befördern könnten. Saeki kam schließlich zu der Erkenntnis, dass die Aufgabe weniger darin bestehe, sich Theorie und Gedankengut des ausländischen Strafrechts, wie des deutschen, anzueignen, sondern vielmehr darin, die dem japanischen Strafrecht eigenen Theorien und Gedanken zu bestärken, sobald das Stadium erreicht sei, in dem die Umstände eine „Errichtung eines dem Großostasiatischen Krieg angemessenen japanischen Strafrechts“ forderten. In Bezug auf Saekis Beteiligung an der Bewegung für japanische Rechtsvernunft lautet Nakayamas würdigende Einschätzung: „Es lässt sich sagen, dass seine Bemühungen einer historischen Analyse des japanischen Rechts und sein Hinweisen auf die Notwendigkeit der Festsetzung eigenständiger Gesichtspunkte bei der Vergleichsmethode hierbei ein positives Nebenprodukt

17) Matsuo (Fn. 15), S. $274 \mathrm{ff}$.

18) Nakayama (Fn. 13), S. 64 ff.

19) Nakayama (Fn. 14), S. 115. In Fußnote 6 wird die Vorstellung des Wirtschaftsstrafrechts (Keizai Keihō) sowie des Sonderstrafrechts in Kriegszeiten (Senji Keiji Tokubetsu Hō) durch Saeki kurz vor dem Krieg angesprochen. 
waren." Was die japanische Bewegung für Rechtsvernunft aber eigentlich anstrebte, war nicht etwa, eine geeignete Strafrechtslehre aus dem Ausland zu kopieren, wie beispielweise die deutsche. Vielmehr vertrat die Bewegung einen Strafrechtsbegriff, der ausschließlich mittels der Erfahrung des japanischen Volkes in Bezug auf Kultur und Geschichte Japans gewonnen werden konnte. Möglicherweise beabsichtigte Saeki also infolgedessen, indem er sich der Erforschung der Tradition des Staatsrechts und der Kokugaku-Schule zuwandte, eine von Geschichte und Tradition gestützte strafrechtliche Mentalität des japanischen Staates herauszuarbeiten, und wurde sich dabei durch seine Analyse der Geschichte des japanischen Rechts sowie mit Hilfe der vergleichenden Forschung zu Rechtssystemen aus verschiedenen Ländern die Eigentümlichkeit des japanischen Strafrechts, dessen Unabhängigkeit von anderen Ländern sowie den besonderen Wert der aus Selbstbestimmung, Nachsicht etc. bestehenden ,,japanischen Tradition der Wahrheit“ gewahr. In diesem Fall kann die im realen japanischen Strafrecht in Erscheinung tretende ,japanische Tradition der Wahrheit“ nicht den japanischen Staat transzendieren und nicht als „ultranationalistisch“ bezeichnet werden. Jedoch kann die ,,japanische Tradition der Wahrheit" auch nicht einfach als ,positives Nebenprodukt“ abgegrenzt und losgelöst von dem „Hauptprodukt“, der Bewegung für japanische Rechtsvernunft, beurteilt werden.

Auch wenn man dies wie Nakayama theoretisch als ,positives Nebenprodukt“ bewerten könnte, muss man sich dennoch die Frage stellen, weshalb Saeki seinerzeit keine effektive Kritik am Hauptprodukt, der Bewegung für japanische Rechtsvernunft, äußerte. Hierin besteht der zweite der von Nakayama angeführten Aspekte. Obwohl Nakayama der Ansicht ist, dass Saekis Analyse des japanischen Rechts ein „positives Nebenprodukt“ hervorgebracht hatte, sieht er Saekis Strafrechtsforschung vor dem Krieg kritisch und zeigt deren Grenzen auf. Hierzu schreibt er: „Der japanischen Bewegung für Rechtsvernunft, und somit auch Dr. Saeki, fehlte eine wesentliche kritische Sicht auf die damalige Situation des japanischen Staates, eine Tendenz zum den freien Individualismus beseitigenden ,Nationalismus“ ist klar ersichtlich,“" und „gerade dieser Aspekt stellte ein Problem dar.“(“20) Angenommen, die ,japanische Tradition der Wahrheit“ sei eine historische Tatsache und gäbe einen Hinweis auf den Idealzustand des Staates oder des Strafrechts; wenn hierin wirklich ein kritischer Gedanke bezüglich des real existierenden Staates oder in Bezug auf das Strafrecht zu finden gewesen war, so hätte daraus, wie Nakayama betont, vielleicht eine kritische Auseinandersetzung mit dem derzeitigen Zustand des japanischen Staates folgen können. Wenn dies noch dazu, selbst als ein theoretisches Produkt, die Idee der Nachsicht oder Bedachtsamkeit des Staates hätte einbringen können, so hätte dies vielleicht in der Realität die Anwendung der Strafgewalt des Staates mildern können. Jedoch handelt es sich bei der ,japanischen Tradition der Wahrheit“ nicht um eine Konzeption, die der derzeitigen Lage des japanischen Staates und Strafrechts auf kritische Weise entgegen-

२0) Nakayama (Fn. 13), S. 65. 
wirken konnte. Die ,japanische Tradition der Wahrheit" betrachtete das reale staatliche Strafrecht nicht kritisch von außen, sie funktionierte vielmehr immanent und dialektisch. Saeki verstand die Auslegung des Strafrechts ebenso wie die Strafgesetzgebung als Manifestation der japanischen Tradition und des eigentümlichen Wesens des japanischen Strafrechts.

Katsuyoshi Ikuta beispielsweise analysierte Saekis Staats- und Menschenbild, welches den gedanklichen Hintergrund seines Strafrechts bildete. ${ }^{21)}$ Nach Ikutas Einschätzung gründete sich Saekis Sicht auf den Staat und das Strafrecht nicht auf eine Idee derselben, sondern auf den existierenden Staat und das bestehende Strafrecht. Mit anderen Worten, Saekis Bild von Staat und Strafrecht gründete sich nicht im Gegensatz zum staatlichen Strafrecht, in dem rechtliche Prinzipien wie das „Naturrecht“ existierten, nicht auf der Ansicht, dass sich das Strafrecht hier in seiner idealen Gestalt zeige, oder dass die Auffassungen von Rechtswidrigkeit und Schuld eines in der Welt des „Naturrechts“ zur Idee gewordenen ,staatlichen Strafrechts in seiner idealen Form“ die Anwendung von staatlichem Strafrecht in der Realität einschränke. Stattdessen wurde es von dem Gedanken getragen, dass der bestehende Staat etwa die durch das Naturrecht hervorgebrachte schöpferische Entdeckung des Rechts oder die Idealisierung von Gesetzen einschränke. Das bedeutet, dass Saeki die Legalität des bestehenden Staates und dessen Strafrecht anerkannte, und darüber hinaus eine Strafrechtslehre vertrat, die davon ausging, dass der real existierende Staat an sich die Möglichkeit habe, das Strafrecht auf selbstbeschränkende Weise auszulegen und anzuwenden. Wenn sich nun Saekis Methode zur Auslegung des Strafrechts am Staate orientierte, so handelt es sich dabei nicht um eine Theorie, die den gegenwärtigen Zustand kritisch beurteilt, indem sie durch Prinzipien wie dem Rückwirkungsverbot, der strafbaren Rechtswidrigkeit und der Zumutbarkeit eine Einschränkung des vom Staate ausgelegten und angewandten Strafrechts oder eine Präzisierung und Eingrenzung von Strafen von Seiten des Volks vorsieht. Es handelt sich vielmehr lediglich um eine Theorie, die die gegenwärtige Situation zu verbessern sucht, indem sie annimmt, der Staat solle schlicht selbst für eine bessere Auslegung des Strafrechts sorgen. Selbstverständlich gibt es auch Fälle, in denen die Auslegung durch den Staat mit den Interessen des Volkes übereinstimmt, aber über den Bereich der „japanischen Tradition der Wahrheit" hinaus würden diese sicher nicht mehr geschützt.

Die ,japanische Tradition der Wahrheit“ diente Saeki auch als Orientierungshilfe zur Beurteilung der Strafgesetzgebung. Beispielweise erklärte Saeki in seiner Darstellung der Strafrechtsgeschichte in Strafrecht Allgemeiner Teil (1941), die Arbeit an einer umfassenden Reform des Strafrechts sei deshalb gescheitert, weil der „Vorläufige Entwurf einer Strafrechtsreform“ („Kaisei Keihō Kari-An“, 1940) eine Nachahmung des deutschen

21) Vgl. Katsuyoshi Ikuta, „Saeki Keihō Riron no Shisō-teki Haikei“ [Saekis Strafrechtstheorie und ihre gedanklichen Hintergründe], in: Keihō Zasshi Bd. 48, Nr. 1 (2008), S. 97. 
Entwurfs und keine eigenständige Schöpfung spezifisch japanischen Strafrechtsdenkens gewesen sei. Überdies argumentierte er, dass mit der Verstärkung des defensiven Charakters des gesamten Strafrechtssystems durch Sondergesetze wie dem Gesetz zur Aufrechterhaltung der Landesverteidigung (Kokubō Hoan Hō, 1941), dem Gesetz zur Aufrechterhaltung der öffentlichen Sicherheit (Chian Iji Hō, 1941) und dem Sonderstrafrecht in Kriegszeiten (Senji Keiji Tokubetsu Hō, 1942) eine bewusste und eigenständige Entwicklung dieses spezifisch japanischen Strafrechtsdenkens zu erwarten sei und es ist anzunehmen, dass die Verteidigung des japanischen Staates als wesentlicher Bestandteil von Tradition und Denken unerlässlich war, um an der ,japanischen Tradition der Wahrheit“ im Strafrecht festhalten zu können. Selbst als der japanische Wehrstaat Vorkehrungen in Form von vielfältigen Sondergesetzgebungen und Kriminalpraktiken zur Erhaltung der öffentlichen Sicherheit traf, um im „Großostasiatischen Krieg“ triumphieren zu können, regte die „japanische Tradition der Wahrheit“ nicht zum Widerstand dagegen an.

Auch wenn die auf dieser Sichtweise auf Staat und Strafrecht basierende Strafrechtslehre Saekis, und insbesondere das Prinzip der strafbaren Rechtswidrigkeit sowie das Zumutbarkeitsprinzip, als Widerstandswaffe oder als Theorie der Befreiung im Strafrecht der Nachkriegszeit fortgeführt und weiterentwickelt werden kann, müssen zuallererst das zugrunde liegende Strafrechtsdenken sowie die entsprechende Methodik einer kritischen Betrachtung unterzogen und ferner die Existenz eines inneren Zusammenhangs mit Prinzipien wie der strafbaren Rechtswidrigkeit sowie deren Umfang überprüft werden. Dies ist jedoch bisher nur in unzureichendem Maße geschehen, und so bleibt nach wie vor eine ganze Reihe von Fragen unbeantwortet. 